\title{
Contribution of postnatal collagen reorientation to depth-dependent mechanical properties of articular cartilage
}

\author{
Mark C. van Turnhout • Sander Kranenbarg • \\ Johan L. van Leeuwen
}

Received: 16 March 2010 / Accepted: 20 May 2010 / Published online: 6 June 2010

(C) The Author(s) 2010. This article is published with open access at Springerlink.com

\begin{abstract}
The collagen fibril network is an important factor for the depth-dependent mechanical behaviour of adult articular cartilage (AC). Recent studies show that collagen orientation is parallel to the articular surface throughout the tissue depth in perinatal animals, and that the collagen orientations transform to a depth-dependent arcade-like structure in adult animals. Current understanding on the mechanobiology of postnatal AC development is incomplete. In the current paper, we investigate the contribution of collagen fibril orientation changes to the depth-dependent mechanical properties of AC. We use a composition-based finite element model to simulate in a 1-D confined compression geometry the effects of ten different collagen orientation patterns that were measured in developing sheep. In initial postnatal life, AC is mostly subject to growth and we observe only small changes in depthdependent mechanical behaviour. Functional adaptation of depth-dependent mechanical behaviour of AC takes place in the second half of life before puberty. Changes in fibril orientation alone increase cartilage stiffness during development through the modulation of swelling strains and osmotic pressures. Changes in stiffness are most pronounced for small stresses and for cartilage adjacent to the bone. We hypothesize that postnatal changes in collagen fibril orientation induce mechanical effects that in turn promote these changes. We further hypothesize that a part of the depth-dependent postnatal increase in collagen content in literature is initiated by the depth-dependent postnatal increase in fibril strain due to collagen fibril reorientation.
\end{abstract}

M. C. van Turnhout $(\varangle)$ · S. Kranenbarg · J. L. van Leeuwen Experimental Zoology Group, Department of Animal Sciences, Wageningen University, PO Box 338, 6700 AH Wageningen, The Netherlands

e-mail: mark.vanturnhout@wur.nl
Keywords Articular cartilage - Collagen network · Development · Depth-dependent properties . Fibril reinforced poroviscoelastic swelling model

\section{Introduction}

Articular cartilage (AC) consists of a hypo-cellular porous extracellular matrix $(\approx 20 \%$ wet weight) that is saturated with fluid ( $\approx 80 \%$ wet weight). The (solid) extracellular matrix is made of mostly collagen $(\approx 80 \%$ dry weight $)$ and the negatively charged proteoglycan matrix $(\approx 20 \%$ dry weight $)$, e.g. Mow and Guo (2002). The relative fractions of these components and the predominant orientation of the collagen fibrils vary over the tissue depth. Experiments have shown that as a result, the mechanical behaviour of healthy adult articular cartilage is depth-dependent (Buckley et al. 2008; Chahine et al. 2007; Chen et al. 2001a; Choi et al. 2007; Erne et al. 2005; Guilak et al. 1995; Gründer 2006; Laasanen et al. 2003; Schinagl et al. 1997; Wong et al. 1997; Zheng et al. 2005). The equilibrium stiffness of AC increases with increasing distance from the articular surface and this is linked to increasing osmotic pressures with increasing distance from the surface (e.g. Klein et al. 2007; Laasanen et al. 2003; Wang et al. 2002; Wong et al. 1997; Wilson et al. 2007). The collagen network is thought to be an important factor for the mechanical behaviour of AC in transient loads (Julkunen et al. 2008b; Korhonen et al. 2003; Laasanen et al. 2003; Li et al. 2009; Shirazi and Shirazi-Adl 2008; Shirazi et al. 2008), but also contributes to the equilibrium stiffness (e.g. Chen et al. 2001b; Korhonen et al. 2002; Laasanen et al. 2003). The collagen network in unloaded healthy adult AC counterbalances the internal osmotic pressures in the tissue, and loss of collagen results in larger swelling strains and therefore smaller osmotic pressures (Bank et al. 2000; Basser et al. 
1998; Maroudas 1976; Maroudas and Venn 1977; Maroudas et al. 1980; Mow and Guo 2002).

The depth-dependent collagen fibril arrangement in adult AC is commonly referred to as 'Benninghoff structure' (Benninghoff 1925; Hughes et al. 2005; Rieppo et al. 2009; Zambrano et al. 1982) and is characterized by three layers: from articular surface to tidemark, there is first a thin layer with collagen fibrils mainly oriented parallel to the articular surface, second there is a thicker transitional zone where the collagen fibrils appear to lack a predominant orientation, and third, there is the deep zone where collagen fibrils are mainly oriented perpendicular to the tidemark. Recent studies in mammals show that the Benninghoff structure is absent at birth and that the predominant orientation of collagen fibrils in perinatal $\mathrm{AC}$ is parallel to the articular surface throughout most of the tissue depth (Julkunen et al. 2009a, 2010; Rieppo et al. 2009; Van Turnhout et al. 2008, 2010).

When we assume that the depth-dependent mechanical properties of adult AC partly reflect the effects of a depth-dependent adult collagen structure (Li et al. 2002a,b; Korhonen and Herzog 2008; Shirazi and Shirazi-Adl 2008; Julkunen et al. 2008a, 2009a), we can expect that the depthdependent mechanical properties manifest less in the AC of perinatal animals that lack differentiation in collagen fibril orientation over the tissue depth. Since collagen loss affects free swelling behaviour of $\mathrm{AC}$, we can expect that a close to $90^{\circ}$ change in collagen orientation in the deep zone during development also affects free swelling behaviour in a similar way. AC stiffness increases during postnatal development (Brommer et al. 2005; Julkunen et al. 2009a; Klein et al. 2007; Williamson et al. 2001; Wong et al. 2000), and the postnatal changes in collagen fibril orientation may contribute to this stiffening.

We cannot rely on experiments with AC from different developmental stages (e.g. Buckley et al. 2009; Klein et al. 2007) if we want to estimate to what degree the postnatal changes in collagen fibril orientation contribute to the (depth-dependent) mechanical behaviour of AC. In such experiments, it will be very hard to separate the effects of changes in collagen orientation from the other developmental effects such as changes in proteoglycan content. With composition-based finite element models, however (e.g. Ateshian et al. 2009; Li et al. 2002b; Korhonen and Herzog 2008; Wilson et al. 2006b), effects of different collagen fibril orientations can be investigated with all other parameters constant. The Fibril Reinforced Poro-Viscoelastic Swelling (FRPVS) model for AC is such a compositional-based model that can model realistic collagen fibril orientations (Wilson et al. 2004, 2005a, 2006b; Julkunen et al. 2007). Wilson et al. (2007) used the FRPVS model to simulate the confined compression experiments on adult bovine AC by Schinagl et al. (1997). They showed that the FRPVS model could capture the depth-dependent mechanical properties as measured by
Schinagl et al. (1997) based on AC composition and structure alone (Wilson et al. 2007).

Our knowledge on the interaction between the mechanical driving mechanism for postnatal AC development and the changing mechanical state due to postnatal AC development is still limited. The postnatal reorientation of collagen fibrils has only recently been described quantitatively (Julkunen et al. 2010; Van Turnhout et al. 2010), and the implications for AC mechanics have not been thoroughly investigated. Such investigations will contribute to our understanding of the mechanobiology of development, maintenance and degeneration of $\mathrm{AC}$, and may be helpful when we try to mimic functional adaptation of $\mathrm{AC}$ in vitro, e.g. in the field of tissue engineering.

In the current paper, we investigate how the mechanical environment changes in postnatal $\mathrm{AC}$ as a result of changes in predominant collagen fibril orientation. We implement recently published data by Van Turnhout et al. (2010) on collagen fibril orientation in developing sheep from birth to maturity in the FRPVS model. We will use the same 1-D confined compression geometry as in Wilson et al. (2007) and investigate how the (depth-dependent) effective aggregate modulus, osmotic pressures and fibril strains change as a result of changes in predominant collagen fibril orientation.

\section{Methods}

For a detailed description of the modelling theory of the FRPVS model, we refer to existing literature (e.g. Julkunen et al. 2007, 2009b; Wilson et al. 2005a, 2006b, 2007). Briefly, this is a biphasic model with the solid phase divided into a viscoelastic fibrillar part that represents the collagen network and a viscoelastic non-fibrillar part that represents the remainder of the extracellular matrix. The model accounts for osmotic pressures due to the negative charges in the solid matrix and strain-dependent permeability and compressibility. Initial fluid fractions, fixed charge densities and collagen fractions and orientations are defined locally (per integration point), and the model accounts for the distinction between intra- and extra-fibrillar fluid when it updates the local composition and mechanical state.

We created ten meshes for the ten developmental stages presented by Van Turnhout et al. (2010). Before the simulations, these meshes differed only in implemented collagen fibril orientations. We used the FRPVS model with depthdependent composition and material parameters as described in Wilson et al. (2007). Thus, with $z$, the dimensionless depth, the initial fluid volume fraction for each mesh was $n_{\mathrm{f}}=0.9-$ $0.2 z$. The initial collagen volume fraction for each mesh was $n_{\text {coll }}=1.4 z^{2}-1.1 z+0.59$. The initial fixed charge density $[\mathrm{Meq} / \mathrm{ml}]$ for each mesh was $c_{\mathrm{F}}=-0.1 z^{2}+0.24 z+0.035$. Collagen density and matrix density were both $1.43 \mathrm{~g} / \mathrm{ml}$, 


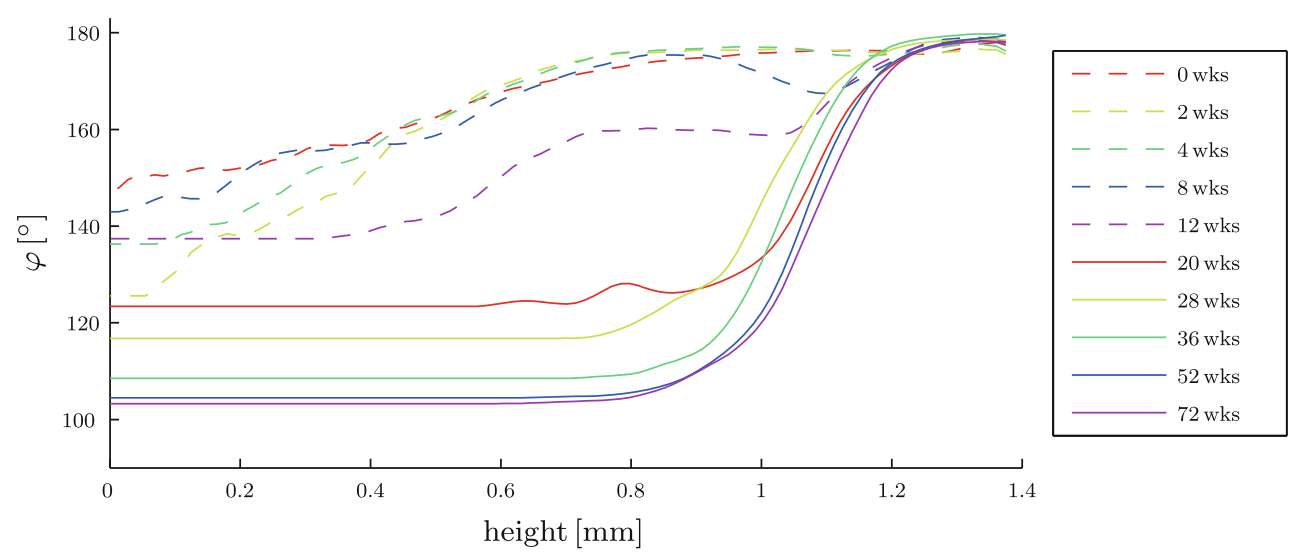

Fig. 1 Fibril directions that were used in this study for the 10 different ages. Adopted from (Van Turnhout et al. 2010, Fig. 3)

for each mesh. Because we are only interested in the equilibrium response, collagen fibrils were modelled as elastic (as opposed to viscoelastic), and permeability was kept constant (as opposed to strain dependent) (Wilson et al. 2007).

We used the original data that was presented by (Van Turnhout et al. 2010, Fig. 3) to simulate the effect of collagen fibril orientation remodelling during development. The patterns for the mean predominant collagen orientation for the ten time points were scaled to equal (dimensionless) length and smoothed with a 5-point moving average. As described by Van Turnhout et al. (2010), the original patterns do not accurately reflect the fact that collagen orientation becomes constant in the deep zone. We therefore kept the deep zone orientation constant once a minimum was encountered. The resulting ten patterns that were used in this study for the ten different ages in Van Turnhout et al. (2010) are collected in Fig. 1. In the simulations, these patterns were modelled with two fibrils in each integration point. One fibril followed the pattern, its companion followed the pattern mirrored in the vertical axis (or mirrored in $90^{\circ}$ in Fig. 1). In this manner, an arcade-like configuration was formed, suitable for 1-D analysis.

The finite element geometry was similar to that described by (Wilson et al. (2007) Section 2.4). The simulations comprised of a 1-dimensional model with a single column of 33 equally thick axisymmetric pore pressure elements (CAX4P), see Fig. 2. Because of the osmosis in the model, the mesh has to be allowed to equilibrate with its environment before the simulation can start at $t_{0}$. The initial mesh height was adapted for each mesh such that the height of each mesh after the initial equilibrium step was $h_{0}=\max h\left(t_{0}\right)=1.375 \mathrm{~mm}$. We prescribed zero pore pressure at the top of the model, i.e. fluid can move freely through this surface. All other surfaces were assumed to be impermeable. Displacements in the radial direction were suppressed. The model was axially compressed in a stepwise manner with

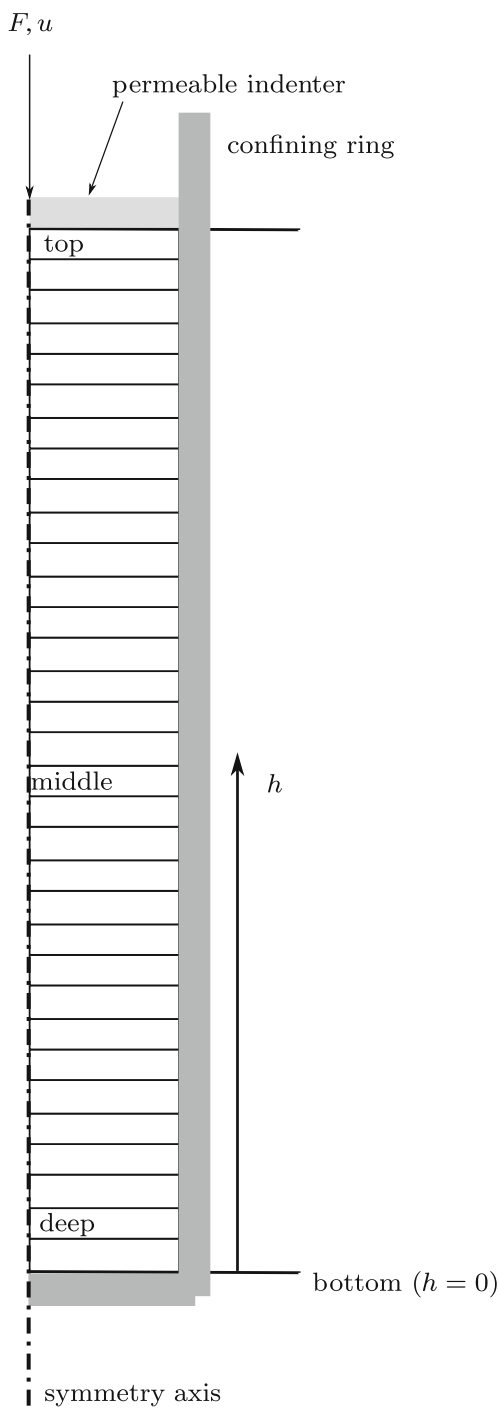

Fig. 2 Mesh for the confined compression simulations. The mesh is divided into 33 elements and is supported by an impermeable bottom and confined by an impermeable ring. The displacement is applied to the permeable indenter at the articular surface. The layers labelled deep, middle and top are used to present the results 
Fig. 3 Mechanical state as a function of mesh height $h$ at the start of the simulation $\left(t_{0}\right)$ for four ages. With: a initial swelling strain $\varepsilon_{i}$, b fibril strain $\varepsilon_{f}, \mathbf{c}$ osmotic pressures $-\Delta \pi$, and $\mathbf{d}$ local effective aggregate modulus $H_{A}(h)$
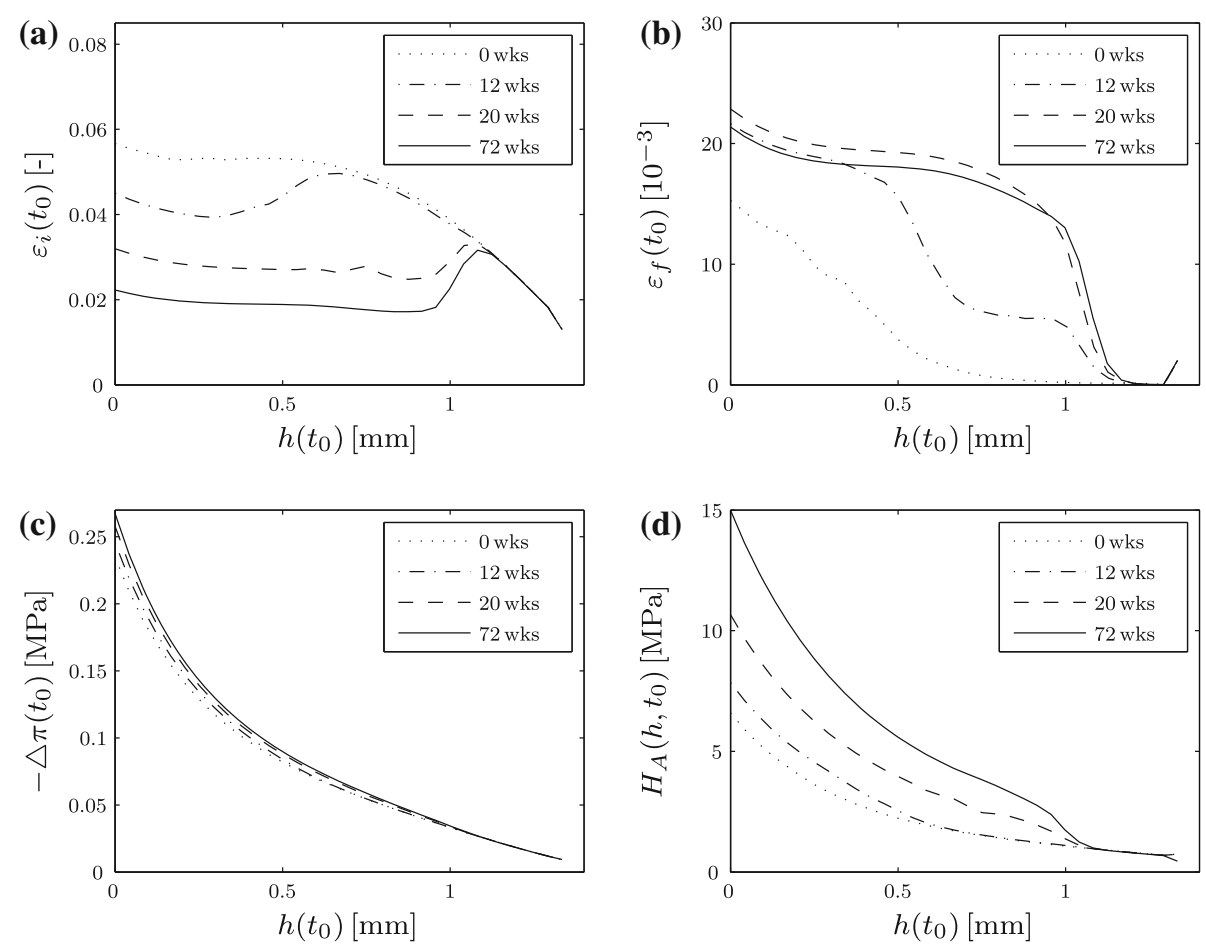

$10^{-3} \%$ strain/second in $1 \%$ intervals, and allowed to reach equilibrium after each compressive step (relaxation).

We used the applied normal stress $\sigma$ and mesh height $h_{0}$ at equilibrium for the computation of global strain $\varepsilon_{g}$ and global effective aggregate modulus $H_{A}$ :

$\varepsilon_{g}=\frac{\max (h)-h_{0}}{h_{0}}, \quad H_{A}=\frac{\partial \sigma}{\partial \varepsilon_{g}}$

with $h_{0}$ the mesh height at the start of the simulation. We used the element thickness $d$ at equilibrium for computation of local strain $\varepsilon_{l}$ and local effective aggregate modulus $H_{A}(h)$ for each element:

$\varepsilon_{l}=\frac{d-d\left(t_{0}\right)}{d\left(t_{0}\right)}, \quad H_{A}(h)=\frac{\partial \sigma}{\partial \varepsilon_{l}}$

with $d\left(t_{0}\right)$ the element thickness at the start of the simulation.

\section{Results}

\subsection{Initial equilibrium}

As expected, the changes in collagen fibril orientations change the mechanical state in the cartilage at $t_{0}$ (Fig. 3; Table 1). The global swelling strain (calculated with Eq. 1) decreases with increasing age (Table $1, \varepsilon_{i}$ ). The changes in depth-dependent swelling strain (calculated with Eq. 2) correlate with changes in depth-dependent fibril orientation (Fig. 3a; Table 1). Between ages, local swelling strains are similar where the fibril orientations are also similar (at the top of the mesh), and changes in local swelling strain are large where the changes in fibril orientations are also large (at the bottom of the mesh).

Depth-dependent changes in fibrils strains at $t_{0}$ also correlate with changes in fibril orientation (Fig. 3b; Table 1, $\varepsilon_{f}$ ). Fibril strains are higher near the bottom of the mesh for all ages, and fibrils strain near the bottom of the mesh increase with increasing age. Between ages, fibrils strains are similar at the top of the mesh. However, contrary to the swelling strains (monotonic increase with age), fibril strains near the bottom of the mesh increase between 0 and 20 weeks, and then show a small decrease to 72 weeks.

Osmotic pressures at $t_{0}$ increase from the top of the mesh to the bottom of the mesh for all ages (Fig. 3c; Table 1, $-\Delta \pi)$. Between ages, $-\Delta \pi$ is similar at the top of the mesh, and there is a gradual increase in $-\Delta \pi$ with depth and age towards the bottom of the mesh.

These changes in the mechanical state at $t_{0}$ (Fig. 3a-c) affect the local effective aggregate modulus $H_{A}(h)$ at $t_{0}$ (Fig. 3d). As with the previous parameters, $H_{A}(h)$ is similar between ages at the top of the mesh. Towards the bottom of the mesh, $H_{A}(h)$ increases with increasing age, and the increase is larger for greater depth (smaller height).

\subsection{Confined compression equilibrium}

The global strain/stress-stiffness relationships show the expected tension-compression nonlinearity (Fig. 4), i.e. first strain softening for small strains, and then strain hardening 
Table 1 Mechanical state for three layers (top, middle and deep) at the start of the simulation $\left(t_{0}\right)$ for all ages

\begin{tabular}{|c|c|c|c|c|c|c|c|c|c|c|c|c|c|}
\hline \multirow[t]{2}{*}{ Age (weeks) } & \multicolumn{4}{|c|}{$\varepsilon_{i}\left(t_{0}\right)\left[10^{-2}\right]$} & \multicolumn{3}{|c|}{$\varepsilon_{f}\left(t_{0}\right)\left[10^{-3}\right]$} & \multicolumn{3}{|c|}{$-\Delta \pi\left(t_{0}\right)[\mathrm{kPa}]$} & \multicolumn{3}{|c|}{$H_{A}\left(h, t_{0}\right)[\mathrm{MPa}]$} \\
\hline & Global & Top & Middle & Deep & Top & Middle & Deep & Top & Middle & Deep & Top & Middle & Deep \\
\hline 0 & 4.68 & 1.30 & 5.18 & 5.55 & 2.01 & 1.68 & 14.3 & 9.21 & 66.3 & 209 & 0.74 & 1.80 & 5.90 \\
\hline 2 & 4.30 & 1.30 & 5.19 & 3.49 & 2.03 & 0.88 & 22.1 & 9.22 & 66.6 & 226 & 0.74 & 1.81 & 8.68 \\
\hline 4 & 4.47 & 1.30 & 5.19 & 4.30 & 2.02 & 1.05 & 21.1 & 9.21 & 66.5 & 219 & 0.74 & 1.81 & 7.20 \\
\hline 8 & 4.62 & 1.30 & 5.18 & 5.18 & 2.00 & 1.73 & 17.4 & 9.21 & 66.4 & 212 & 0.74 & 1.80 & 6.19 \\
\hline 12 & 4.08 & 1.30 & 4.95 & 4.36 & 2.01 & 8.95 & 20.9 & 9.22 & 67.1 & 219 & 0.74 & 1.87 & 7.10 \\
\hline 20 & 2.78 & 1.30 & 2.69 & 3.09 & 2.01 & 18.8 & 22.1 & 9.23 & 71.2 & 229 & 0.66 & 3.19 & 9.68 \\
\hline 28 & 2.59 & 1.30 & 2.28 & 2.66 & 2.01 & 18.5 & 21.6 & 9.24 & 72.1 & 233 & 0.64 & 3.74 & 11.1 \\
\hline 36 & 2.27 & 1.30 & 1.97 & 2.30 & 2.01 & 17.9 & 21.0 & 9.24 & 72.7 & 236 & 0.51 & 4.31 & 12.8 \\
\hline 52 & 2.13 & 1.30 & 1.87 & 2.18 & 2.01 & 17.7 & 20.7 & 9.24 & 72.8 & 237 & 0.46 & 4.52 & 13.5 \\
\hline 72 & 2.08 & 1.30 & 1.85 & 2.15 & 2.01 & 17.7 & 20.6 & 9.24 & 72.9 & 237 & 0.45 & 4.58 & 13.7 \\
\hline
\end{tabular}

With: $\varepsilon_{i}$-initial swelling strain, $\varepsilon_{f}$-fibril strain, $-\Delta \pi$-osmotic pressures, and $H_{A}(h)$-local effective aggregate modulus

Fig. 4 Global effective aggregate modulus $H_{A}$ as a function of a global strain $\varepsilon_{g}$, and $\mathbf{b}$ global stress $\sigma$
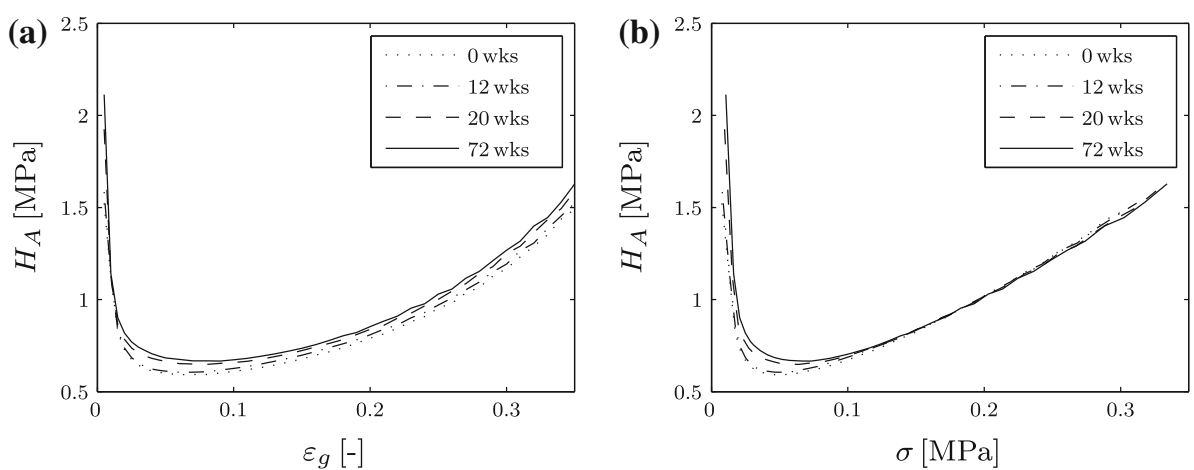

for larger strains (Fig. 4a). The transition between strain softening and strain hardening shifts towards larger global strain/stress between 0 weeks (minimum $H_{A}$ at $0.05 \%$ / $40 \mathrm{kPa}$ ) and 72 weeks (minimum $H_{A}$ at $0.08 \% / 67 \mathrm{kPa}$ ). $H_{A}$ is larger for increasing age for all simulated global strains (Fig. 4a), and for global stresses up to $\approx 0.15 \mathrm{MPa}$ (Fig. 4b). The increase in $H_{A}$ between ages is largest for small strains/stresses.

The local effective aggregate modulus $H_{A}(h)$ in compression is similar between ages for cartilage layers that are past their (local) tension-compression transition, i.e. that are loaded in compression (Fig. 5a-c; Table 2). For the (deep) cartilage layers that are still loaded in tension, $H_{A}(h)$ decreases with increasing strain, and $H_{A}(h)$ remains larger for increasing ages. The local strains $\varepsilon_{l}$ change between ages (Fig. 5d-f). For a large range of global strains $\left(\varepsilon_{g} \leq 20 \%\right)$, local strains increase near the top of the mesh, and decrease near the bottom of the mesh for increasing ages (Fig. 5d,e). Local strains become similar between ages for large global strains (Fig. 5f). The location of the tension-compression boundary is at a smaller height for increasing ages, although the differences are small (Fig. 5a-f).
The osmotic pressures increase over the entire cartilage depth during compression (Fig. 5g-i). At small global strains, osmotic pressures are similar between ages at the top of the model (Fig. 5g; Table 1). For larger strains, osmotic pressures become higher for increasing ages over the entire cartilage depth (Fig. 5h,i)

The fibril strains at the top of the model become zero during compression (Fig. 5j-1). At 10\% global strain, fibril strains are zero in the upper half of the model (Fig. 5j), and the region with zero fibril strain increases with increasing global strain (Fig. $5 \mathrm{k}, \mathrm{l}$ ). The pattern that we observed at $t_{0}$, an increase in fibril strain up to age 20 weeks and next a small decrease in fibril strain up to 72 weeks (Fig. 3b), is still present at $10 \%$ global strain (Fig. 5j). For larger strains, changes occur. At $20 \%$ global strain, the age for the maximum fibril strains is 12 weeks (Fig. 5k) and the difference between 0 and 72 weeks is much smaller than at smaller strains (Figs. $3 \mathrm{~b}$ and $5 \mathrm{j})$. At very large strains, fibril strains are lower for the animals of 72 weeks than for the animals of 0 weeks (Fig. 51).

We finally present the local strain-stiffness relationships for the three layers top, middle and deep for four ages (Fig. 6). The top layer is subjected to compression for local strains 
(a)

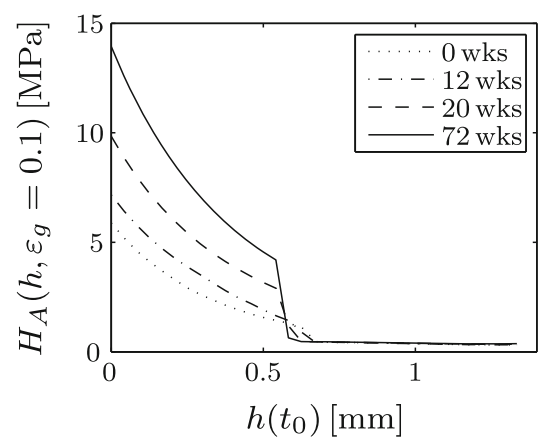

(d)

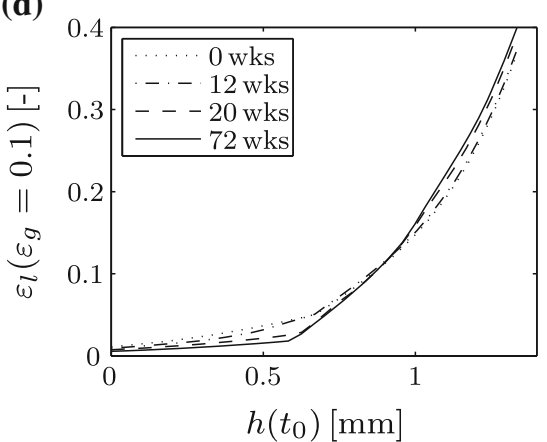

(g)

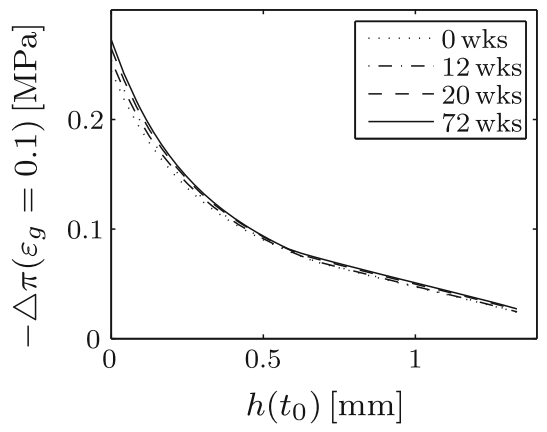

(j)

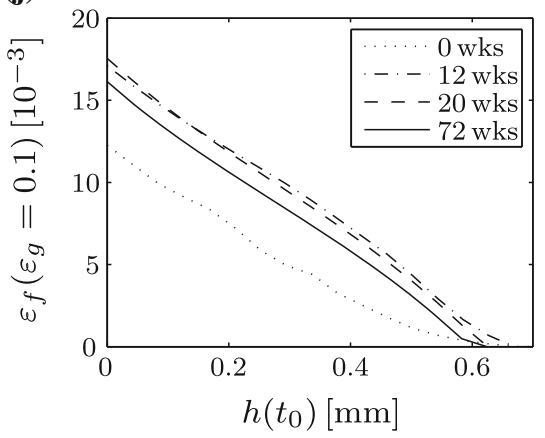

(b)

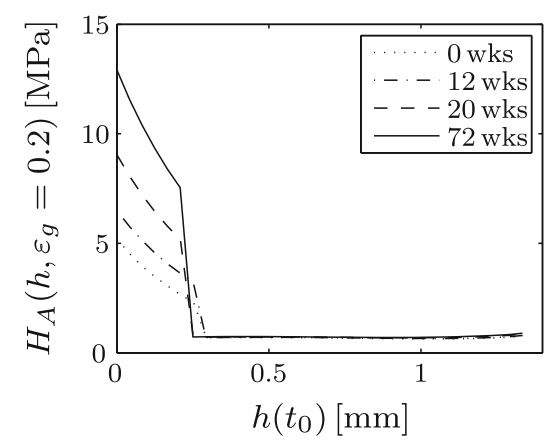

(e)

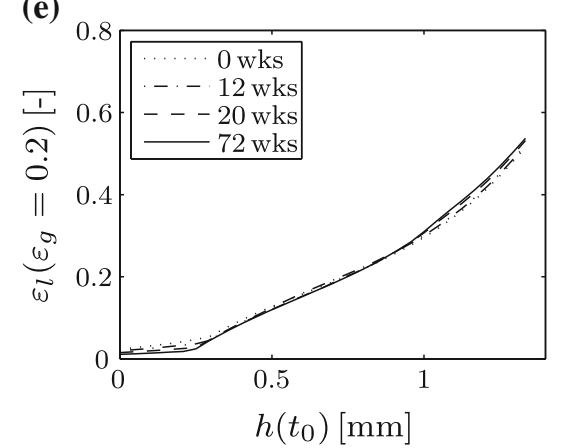

(h)

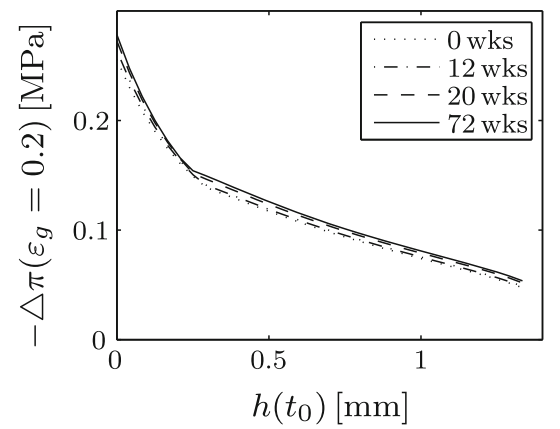

(k)

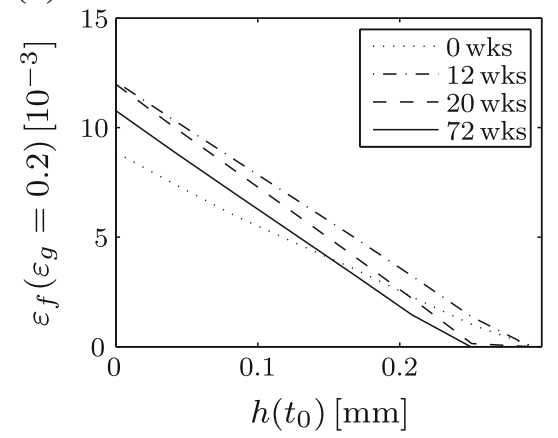

(c)

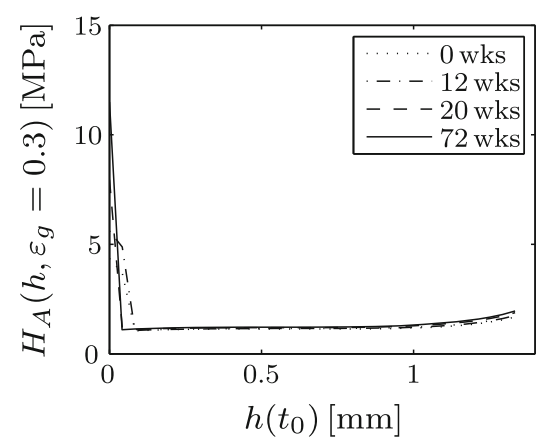

(f)

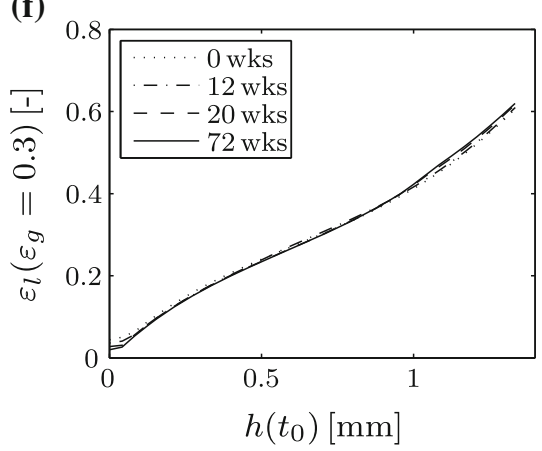

(i)

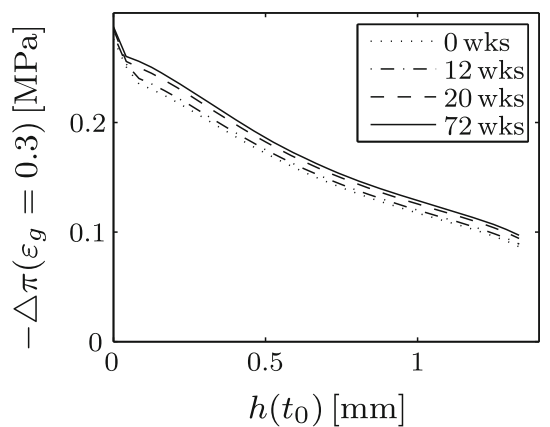

(I)

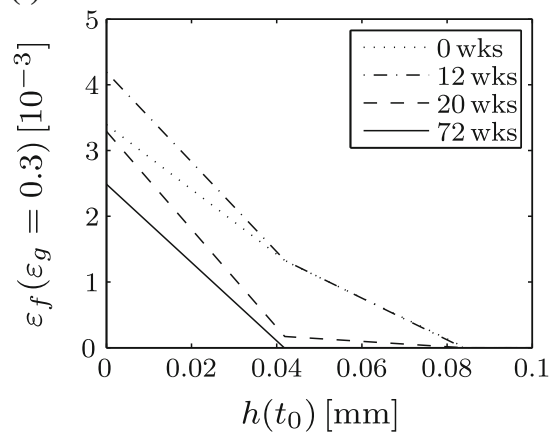

Fig. 5 Mechanical state as a function of mesh height $h$ at three levels of global strain $\varepsilon_{g}$ for four ages. With: a-c-local effective aggregate modulus $H_{A}(h)$ at a $\varepsilon_{g}=10 \%$, b $\varepsilon_{g}=20 \%$, and $\mathbf{c} \varepsilon_{g}=30 \%$; e-g local strain $\varepsilon_{l}$ at $\mathbf{e} \varepsilon_{g}=10 \%$, f $\varepsilon_{g}=20 \%$, and $\mathbf{g} \varepsilon_{g}=30 \%$; $\mathbf{h}-\mathbf{j}$ osmotic pressures $-\Delta \pi$ at $\mathbf{h} \varepsilon_{g}=10 \%$, i $\varepsilon_{g}=20 \%$, and $\mathbf{j} \varepsilon_{g}=30 \%$; and $\mathbf{j}-\mathbf{l}$ local fibril strain $\varepsilon_{f}$ at $\mathbf{a} \varepsilon_{g}=10 \%$, b $\varepsilon_{g}=20 \%$, and $\mathbf{c} \varepsilon_{g}=30 \%$. Note the differences in scale for $h\left(t_{0}\right)$ in panels $(\mathbf{j}-\mathbf{l})$ 

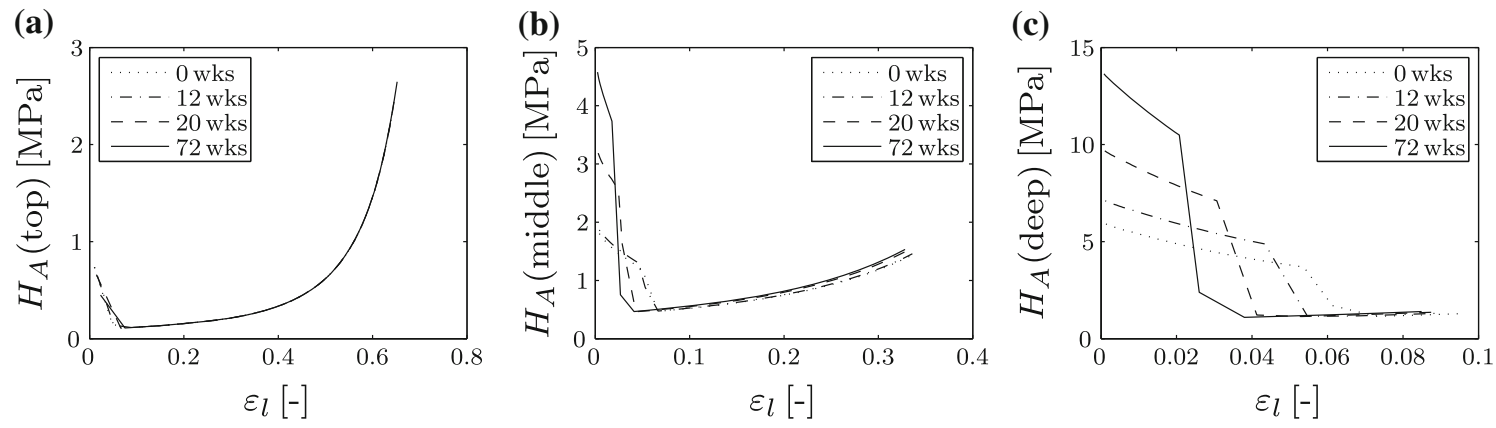

Fig. 6 Local effective aggregate modulus $H_{A}(h)$ as a function of local strain $\varepsilon_{l}$ for three layers in de model: a top, b middle, and $\mathbf{c}$ deep

Table 2 Local effective aggregate modulus $H_{A}(h)$ [MPa] for three layers (top, middle and deep) in the simulations at three levels of global strain $(10,20$ and $30 \%)$ for all ages

\begin{tabular}{|c|c|c|c|c|c|c|c|c|c|c|c|}
\hline & & 0 week & 2 weeks & 4 weeks & 8 weeks & 12 weeks & 20 weeks & 28 weeks & 36 weeks & 52 weeks & 72 weeks \\
\hline \multirow[t]{3}{*}{$\varepsilon_{g}=10 \%$} & Top & 0.31 & 0.32 & 0.32 & 0.31 & 0.32 & 0.35 & 0.36 & 0.36 & 0.37 & 0.37 \\
\hline & Middle & 1.07 & 1.01 & 1.04 & 1.06 & 0.88 & 0.46 & 0.46 & 0.47 & 0.47 & 0.47 \\
\hline & Deep & 5.20 & 7.97 & 6.53 & 5.51 & 6.43 & 8.86 & 10.23 & 11.81 & 12.43 & 12.60 \\
\hline \multirow[t]{3}{*}{$\varepsilon_{g}=20 \%$} & Top & 0.76 & 0.77 & 0.77 & 0.76 & 0.79 & 0.85 & 0.86 & 0.88 & 0.89 & 0.89 \\
\hline & Middle & 0.69 & 0.69 & 0.69 & 0.69 & 0.70 & 0.71 & 0.72 & 0.72 & 0.73 & 0.73 \\
\hline & Deep & 4.53 & 7.25 & 5.87 & 4.86 & 5.76 & 8.06 & 9.35 & 10.83 & 11.42 & 11.58 \\
\hline \multirow[t]{3}{*}{$\varepsilon_{g}=30 \%$} & Top & 1.68 & 1.72 & 1.71 & 1.69 & 1.75 & 1.88 & 1.90 & 1.93 & 1.95 & 1.95 \\
\hline & Middle & 1.13 & 1.15 & 1.14 & 1.13 & 1.16 & 1.19 & 1.20 & 1.21 & 1.21 & 1.22 \\
\hline & Deep & 3.69 & 6.27 & 5.00 & 4.03 & 4.88 & 1.22 & 1.09 & 1.10 & 1.10 & 1.10 \\
\hline
\end{tabular}

larger than $10 \%$ and the $\varepsilon_{l}-H_{A}(h)$ relationship is similar for all ages (Fig. 6a). Changes occur for the $\varepsilon_{l}-H_{A}(h)$ relationships for different ages for layers at a larger depth (Fig. 6b,c). Three observations should be noted: (1) $H_{A}(h)$ remains larger near the bottom of mesh for increasing ages, (2) the tension-compression transition shifts towards smaller local strains for deeper layers, and (3) these effects are larger for deeper layers.

\section{Discussion}

To evaluate the contribution of postnatal collagen orientation development, we performed ten simulations with the composition based FRPVS model which differed only in predominant collagen fibril orientation. And we used measurements on collagen fibril orientation in developing sheep between birth (0 weeks) and maturity (72 weeks) (Van Turnhout et al. 2010) for the collagen orientations.

The differences in local swelling strain after the initial equilibrium step, $\varepsilon_{i}$, in these simulations are the result of the differences in collagen fibril orientation only, as all other parameters were constant. For $h>1.12 \mathrm{~mm}$ (the 'superficial layer'), collagen orientations are similar between the ten meshes (Fig. 1), and this is reflected in Table 1; Fig. 3:
$\varepsilon_{i},-\Delta \pi, H_{A}(h)$ and $\varepsilon_{f}$ are similar for the top layer for all ten simulations. As expected, the changing fibril orientations for $h<1.12$ have a direct effect on $\varepsilon_{i}$ (Fig. 3a; Table 1, $\varepsilon_{i}$ ), and they thereby indirectly influence the local osmotic pressures $-\Delta \pi$ (Fig. 3c; Table 1). These osmotic pressures are known to be an important factor for the equilibrium stiffness of AC (e.g. Basser et al. 1998; Chen et al. 2001b; Laasanen et al. 2003; Mow and Guo 2002). Our simulations show that equal osmotic pressures in the middle and deep zone for simulations for increasing ages, result in increasing values for $H_{A}(h)\left(\varepsilon_{g}=0\right)$ for increasing ages (Fig. 3; Table 1). Thus, the direct effect of changing fibril orientations (smaller $\varepsilon_{i}$ ) and the indirect effect of changing fibril orientations (larger $-\Delta \pi$ ) interact to increase AC stiffness.

The changing fibril orientations affect $H_{A}(h)$ over the entire region of simulated strains and change the tensioncompression behaviour (Fig. 6). Initially, the AC is subject to swelling and thus under tensile stress (Fig. 3a). With increasing global stress, we reduce the amount of swelling until the global stress equals the osmotic pressures. From that point on, a further increase in global stress subjects the $\mathrm{AC}$ to compression and compressive stress (Wilson et al. 2007). $H_{A}$ decreases with the reduction in tension, and $H_{A}(h)$ increases with an increase in compression (Figs. 4 and 6), and the local strain for the tension-compression transition 
becomes smaller with increasing age (Fig. 6). Combined with an increase in $H_{A}(h)$ at zero strain for increasing age (Fig. 3d), this results in higher gradients for the tension behaviour of $H_{A}(h)$ as a function of $\varepsilon_{l}$ for deeper layers (Fig. 6). Although the tension-compression transition shifts to lower $\varepsilon_{l}$ for deeper layers with increasing age (Fig. 6), the transition is associated with higher osmotic pressures for deeper layers with increasing age, i.e. with larger global stresses. Thus, the deeper layers are in fact subject to tension, and therefore retain their high pre-compression $H_{A}(h)$, for an increasing range of global stresses for increasing ages.

The sheep that were used to obtain the experimental data on collagen orientations reached puberty around the age of 36 weeks (Van Turnhout et al. 2010). Figure 1 shows that the remodelling of predominant collagen orientation is mostly done by that age, and that the largest difference in (deep zone) collagen orientation is that between 12 and 20 weeks. In our simulations, we also find the largest effects between 12 and 20 weeks. This is also the age span with the largest rate of change for $\varepsilon_{i}, \varepsilon_{f},-\Delta \pi$ and $H_{A}(h)$ (not shown). Consequently, our simulations show that the changes in depth-dependent mechanical properties are nearly finished at 36 weeks of age (e.g. Tables 1 and 2). Cartilage thickness decreases during postnatal development and Van Turnhout et al. (2010) estimated a time constant of -0.017 weeks for the cartilage thickness in their data. This means that $73 \%$ of the decrease in cartilage thickness occurs before 12 weeks of age, $89 \%$ occurs before 20 weeks of age and cartilage thickness is almost stabilised at 36 weeks of age when $98 \%$ of the decrease in cartilage thickness has occurred. The temporal patterns of collagen fibril reorientation and decrease in cartilage thickness suggest that the changes that we see in AC in the first half of postnatal life to puberty are more related to growth mechanisms (largest decrease in thickness), while the second half of postnatal life to puberty is used for the depth-dependent functional adaptation of $\mathrm{AC}$ (largest changes in collagen orientation and consequently mechanical properties). This is in line with the remark by Hunziker et al. $(2007 a, b)$ that cartilage may only then begin to assume a more mature anisotropic morphological structure as the shaping process of the joint approaches completion.

These simulations do not provide a full description of postnatal AC development. The influence of the collagen network on transient mechanical behaviour of AC (Federico and Herzog 2008a,b; Laasanen et al. 2003) for instance, is also a factor for the mechanical state that drives postnatal $\mathrm{AC}$ development and as such deserves attention. The depthdependent increase in collagen content is also a factor, as are (depth-dependent) changes in FCD and fluid fraction. Also, the simulated geometry was limited to 1-D confined compression. Within these limitations, our simulations provide further insight into postnatal mechanobiology of AC.
They show that analysis of experiments on depth-dependent mechanical properties of premature AC (Klein et al. 2007; Wang et al. 2002; Williamson et al. 2001) should also take the changing fibril orientations into account. An increase in collagen content itself for instance, is associated with an increase in osmotic pressures through the modulation of intra- and extra-fibrillar fluid: collagen binds fluid that is therefore no longer available for osmosis, and this increases the effective FCD (Maroudas and Bannon 1981; Maroudas et al. 1991; Wilson et al. 2007). The increase in collagen content and its influence on osmotic pressure is cited by Williamson et al. (2001) as an explanation for the increase of $180 \%$ in confined compression modulus from foetal to adult bovine AC. Our results show that a part of this $180 \%$ can be attributed to changes in collagen orientation between foetal and adult AC. These changes may also help to roughly predict temporal and spatial patterns of postnatal changes such as collagen content increase and FCD increase and possibly the development of e.g. functional interstitial fluid support (Krishnan et al. 2003; Ateshian 2009) or the depth-dependent phenotype of adult chondrocytes (e.g. Aydelotte and Kuettner 1988; Aydelotte et al. 1988; Korhonen and Herzog 2008; Wu and Herzog 2006).

The simulations in this paper do not clarify why the predominant collagen orientation in the deep zone changes. Based on Wilson et al. (2006a) who showed that principal strains in the matrix are a good predictor for collagen fibril orientation in $\mathrm{AC}$, we hypothesise that (vertical) swelling strains are responsible. The bottom layer of AC is subjected to vertical swelling strains for almost the entire regime of physiological strains in our simulations, contrary to the upper layers (Fig. 5j,k). The current depth-dependent mechanical behaviour for the simulations for the young animals in this paper is mostly due to our choice for depth-dependent composition (i.e. equal to that of the adult). For our hypothesis to hold, depth-dependent mechanical behaviour needs to be present in the AC of perinatal animals. This is not unlikely, since Klein et al. (2007) showed that both proteoglycan content and collagen content increased with depth for foetal and newborn bovine cartilage. Also, experimental results on the mechanics of foetal (Klein et al. 2007) and neonatal (Buckley et al. 2009) AC show that depth-dependent mechanical behaviour is indeed present in perinatal AC.

Two mechanisms affect the initial swelling strain in our hypothesis during postnatal collagen reorientation. First, a portion of the load is transferred from the matrix to the collagen fibrils (Fig. 3a,b), leading to lower swelling strains and higher strains in the collagen fibrils during development. Since we propose that swelling strains initiate deep zone collagen reorientation, this effect reduces the driving mechanism in our hypothesis. Second however, osmotic pressures increase (Fig. 3c) because the decreasing swelling strain 
reduces the free fluid available for osmosis (Bank et al. 2000; Basser et al. 1998; Maroudas 1976; Maroudas and Venn 1977; Maroudas et al. 1980; Mow and Guo 2002). The rise in osmotic pressure in turn affects the matrix and fibril strains in compression: the initial swelling state (AC loaded in tension) is maintained for a larger range of compressive stresses (Fig. 5j,k). Thus, although the swelling strains in our hypothesis become smaller during development, these swelling strains persist for a larger range of global stresses. And this persistence of swelling strains under physiological loads in turn reinforces the driving mechanism for the development of a Benninghoff structure under our hypotheses.

The increased strains during development in the fibrils in the deep zone also persist for larger global stresses. Rieppo et al. (2009) found that collagen content increases during development, and that it increases more in the deep zones of the AC than at the surface of the AC. Based on our simulations, we suggest that this differentiation in collagen content increase is partly initiated by the differentiation in fibril strain. However, if the increase of collagen content in the deep zone is related to the higher fibril strains, two mechanisms are expected to further affect osmotic pressures and fibril strains. First, osmotic pressures and therefore fibril strains will increase through the modulation of intra- and extra-fibrillar fluid content. Second, fibril strains will be less when more collagen is present for given osmotic pressures and orientations. Results of different simulations with the current geometry (results not shown) indicate that when we take both mechanisms into account, osmotic pressures increase and initial matrix and fibril strains (in the deep zone) are indeed a little lower when collagen is added (and everything else is kept equal). As in the previous paragraph, this state also persists for a larger range of compressive stresses due to the rise in osmotic pressures.

Acknowledgments The authors are grateful to Dr. Wouter Wilson and Dr. René van Donkelaar at the Materials Technology group of the Faculty of Biomedical Engineering of Eindhoven University of Technology for the use of, and support with, the FRPVS model.

Open Access This article is distributed under the terms of the Creative Commons Attribution Noncommercial License which permits any noncommercial use, distribution, and reproduction in any medium, provided the original author(s) and source are credited.

\section{References}

Ateshian GA (2009) The role of interstitial fluid pressurization in articular cartilage lubrication. J Biomech. doi:10.1016/j.jbiomech.2009. 04.040

Ateshian GA, Rajan V, Chahine NO, Canal CE, Hung CT (2009) Modeling the matrix of articular cartilage using a continuous fiber angular distribution predicts many observed phenomena. J Biomech Eng 131(6):061,003. doi:10.1115/1.3118773
Aydelotte MB, Kuettner KE (1988) Differences between sub-populations of cultured bovine articular chondrocytes. I. Morphology and cartilage matrix production. Connect Tissue Res 18(3):205-222

Aydelotte MB, Greenhill RR, Kuettner KE (1988) Differences between sub-populations of cultured bovine articular chondrocytes. II. Proteoglycan metabolism. Connect Tissue Res 18(3):223-234

Bank RA, Soudry M, Maroudas A, Mizrahi J, TeKoppele JM (2000) The increased swelling and instantaneous deformation of osteoarthritic cartilage is highly correlated with collagen degradation. Arthritis Rheum 43(10):2202-2210. doi:3.0.CO;2-E

Basser PJ, Schneiderman R, Bank RA, Wachtel E, Maroudas A (1998) Mechanical properties of the collagen network in human articular cartilage as measured by osmotic stress technique. Arch Biochem Biophys 351(2):207-219. doi:10.1006/abbi.1997.0507

Benninghoff A (1925) Form und Bau der Gelenkknorpel in ihren Beziehungen zur Funktion. Zweiter Teil: Der Aufbau des Gelenkknorpels in seinen Beziehungen zur Funktion. ZZellforsch Mikrosk Anat 2:783-862

Brommer H, Brama P, Laasanen M, Helminen H, Van Weeren P, Jurvelin J (2005) Functional adaptation of articular cartilage from birth to maturity under the influence of loading: a biomechanical analysis. Equine Vet J 37(2):148-154

Buckley MR, Gleghorn JP, Bonassar LJ, Cohen I (2008) Mapping the depth dependence of shear properties in articular cartilage. J Biomech 41(11):2430-2437. doi:10.1016/j.jbiomech.2008.05. 021

Buckley MR, Bergou AJ, Fouchard J, Bonassar LJ, Cohen I (2009) High-resolution spatial mapping of shear properties in cartilage. J Biomech. doi:10.1016/j.jbiomech.2009.10.012

Chahine N, Ateshian G, Hung C (2007) The effect of finite compressive strain on chondrocyte viability in statically loaded bovine articular cartilage. Biomech Model Mechanobiol 6(1-2):103-111. doi:10. 1007/s10237-006-0041-2

Chen A, Bae W, Schinagl R, Sah R (2001a) Depth- and straindependent mechanical and electromechanical properties of full-thickness bovine articular cartilage in confined compression. J Biomech 34(1):1-12. doi:10.1016/S0021-9290(00)00170-6

Chen S, Falcovitz Y, Schneiderman R, Maroudas A, Sah R (2001b) Depth-dependent compressive properties of normal aged human femoral head articular cartilage: relationship to fixed charge density. Osteoarthritis Cartilage 9(6):561-569. doi:10.1053/joca. 2001.0424

Choi JB, Youn I, Cao L, Leddy HA, Gilchrist CL, Setton LA, Guilak $\mathrm{F}$ (2007) Zonal changes in the three-dimensional morphology of the chondron under compression: the relationship among cellular, pericellular, and extracellular deformation in articular cartilage. J Biomech 40(12):2596-2603. doi:10.1016/j.jbiomech.2007.01. 009

Erne OK, Reid JB, Ehmke LW, Sommers MB, Madey SM, Bottlang M (2005) Depth-dependent strain of patellofemoral articular cartilage in unconfined compression. J Biomech 38(4):667-672. doi:10.1016/j.jbiomech.2004.04.005

Federico S, Herzog W (2008a) On the anisotropy and inhomogeneity of permeability in articular cartilage. Biomech Model Mechanobiol 7(5):367-378. doi:10.1007/s10237-007-0091-0

Federico S, Herzog W (2008b) On the permeability of fibre-reinforced porous materials. Int J Solids Struct 45(7-8):2160-2172. doi:10. 1016/j.ijsolstr.2007.11.014

Gründer W (2006) MRI assessment of cartilage ultrastructure. NMR Biomed 19(7):855-876. doi:10.1002/nbm. 1092

Guilak F, Ratcliffe A, Mow VC (1995) Chondrocyte deformation and local tissue strain in articular cartilage: a confocal microscopy study. J Orthop Res 13(3):410-421. doi:10.1002/jor.1100130315

Hughes L, Archer C, Ap Gwynn I (2005) The ultrastructure of mouse articular cartilage: collagen orientation and implications for tissue 
functionality. A polarised light and scanning electron microscope study and review. Eur Cell Mater 9:68-84

Hunziker E, Kapfinger E, Geiss J (2007a) Corrigendum to "The structural architecture of adult mammalian articular cartilage evolves by a synchronized process of tissue resorption and neoformation during postnatal development" [Osteoarthritis Cartilage 15(2007) 403-413]. Osteoarthritis Cartilage doi:10.1016/j.joca. 2007.06.001

Hunziker E, Kapfinger E, Geiss J (2007b) The structural architecture of adult mammalian articular cartilage evolves by a synchronized process of tissue resorption and neoformation during postnatal development. Osteoarthritis Cartilage 15(4):403-413. doi:10.1016/j.joca.2006.09.010, corrigendum in Hunziker et al. (2007a)

Julkunen P, Kiviranta P, Wilson W, Jurvelin JS, Korhonen RK (2007) Characterization of articular cartilage by combining microscopic analysis with a fibril-reinforced finite-element model. J Biomech 40(8):1862-1870. doi:10.1016/j.jbiomech.2006.07. 026

Julkunen P, Korhonen R, Nissi M, Jurvelin J (2008a) Mechanical characterization of articular cartilage by combining magnetic resonance imaging and finite-element analysis: a potential functional imaging technique. Phys Med Biol 53(9):2425-2438. doi:10. 1088/0031-9155/53/9/014

Julkunen P, Wilson W, Jurvelin JS, Rieppo J, Qu CJ, Lammi MJ, Korhonen RK (2008b) Stress-relaxation of human patellar articular cartilage in unconfined compression: prediction of mechanical response by tissue composition and structure. J Biomech 41(9):1978-1986. doi:10.1016/j.jbiomech.2008.03.026

Julkunen P, Harjula T, Iivarinen J, Marjanen J, Seppänen K, Närhi T, Arokoski J, Lammi MJ, Brama P, Jurvelin J, Helminen H (2009a) Biomechanical, biochemical and structural correlations in immature and mature rabbit articular cartilage. Osteoarthritis Cartilage 17(12):1628-1638. doi:10.1016/j.joca.2009.07.002

Julkunen P, Jurvelin JS, Isaksson H (2009b) Contribution of tissue composition and structure to mechanical response of articular cartilage under different loading geometries and strain rates. Biomech Model Mechanobiol. doi:10.1007/ s10237-009-0169-y

Julkunen P, Iivarinen J, Brama P, Arokoski J, Jurvelin J, Helminen $\mathrm{H}$ (2010) Maturation of collagen fibril network structure in tibial and femoral cartilage of rabbits. Osteoarthritis \& Cartilage 18(3):406-415. doi:10.1016/j.joca.2009.11.007

Klein TJ, Chaudhry M, Bae WC, Sah RL (2007) Depth-dependent biomechanical and biochemical properties of fetal, newborn, and tissue-engineered articular cartilage. J Biomech 40(1):182-190. doi:10.1016/j.jbiomech.2005.11.002

Korhonen R, Laasanen M, Töyräs J, Rieppo J, Hirvonen J, Helminen H, Jurvelin J (2002) Comparison of the equilibrium response of articular cartilage in unconfined compression, confined compression and indentation. J Biomech 35(7):903-909. doi:10.1016/ S0021-9290(02)00052-0

Korhonen RK, Herzog W (2008) Depth-dependent analysis of the role of collagen fibrils, fixed charges and fluid in the pericellular matrix of articular cartilage on chondrocyte mechanics. J Biomech 41(2):480-485. doi:10.1016/j.jbiomech.2007.09.002

Korhonen RK, Laasanen MS, Töyräs J, Lappalainen R, Helminen HJ, Jurvelin JS (2003) Fibril reinforced poroelastic model predicts specifically mechanical behavior of normal, proteoglycan depleted and collagen degraded articular cartilage. J Biomech 36(9):13731379. doi:S0021-9290(03)00069-1

Krishnan R, Park S, Eckstein F, Ateshian GA (2003) Inhomogeneous cartilage properties enhance superficial interstitial fluid support and frictional properties, but do not provide a homogeneous state of stress. J Biomech Engin 125(5):569-577. doi:10.1115/1. 1610018
Laasanen M, Töyräs J, Korhonen R, Rieppo J, Saarakkala S, Nieminen M, Hirvonen J, Jurvelin J (2003) Biomechanical properties of knee articular cartilage. Biorheology 40(1-3):133-140

Li L, Buschmann M, Shirazi-Adl A (2002a) The role of fibril reinforcement in the mechanical behavior of cartilage. Biorheology 39(1-2):89-96

Li L, Shirazi-Adl A, Buschmann M (2002b) Alterations in mechanical behaviour of articular cartilage due to changes in depth varying material properties - a nonhomogeneous poroelastic model study. Comput Methods Biomech Biomed Engin 5(1):45-52. doi:10. 1080/10255840290008088

Li L, Cheung J, Herzog W (2009) Three-dimensional fibril-reinforced finite element model of articular cartilage. Med Biol Eng Comput 47(6):607-615. doi:10.1007/s11517-009-0469-5

Maroudas A (1976) Balance between swelling pressure and collagen tension in normal and degenerate cartilage. Nature 260(5554):808-809. doi:10.1038/260808a0

Maroudas A, Bannon C (1981) Measurement of swelling pressure in cartilage and comparison with the osmotic pressure of constituent proteoglycans. Biorheology 18(3-6):619-632

Maroudas A, Venn M (1977) Chemical composition and swelling of normal and osteoarthrotic femoral head cartilage. II. Swelling. Ann Rheum Dis 36(5):399-406

Maroudas A, Bayliss M, Venn M (1980) Further studies on the composition of human femoral head cartilage. Ann Rheum Dis 39(5):514 523

Maroudas A, Wachtel E, Grushko G, Katz E, Weinberg P (1991) The effect of osmotic and mechanical pressures on water partitioning in articular cartilage. Biochim Biophys Acta 1073(2):285-294. doi:10.1016/0304-4165(91)90133-2

Mow VC, Guo XE (2002) Mechano-electrochemical properties of articular cartilage: their inhomogeneities and anisotropies. Annu Rev Biomed Eng 4:175-209. doi:10.1146/annurev.bioeng. 4.110701.120309

Rieppo J, Hyttinen M, Halmesmaki E, Ruotsalainen H, Vasara A, Kiviranta I, Jurvelin J, Helminen H (2009) Changes in spatial collagen content and collagen network architecture in porcine articular cartilage during growth and maturation. Osteoarthritis Cartilage 17(4):448-455. doi:10.1016/j.joca.2008.09.004

Schinagl RM, Gurskis D, Chen AC, Sah RL (1997) Depth-dependent confined compression modulus of full-thickness bovine articular cartilage. J Orthop Res 15(4):499-506. doi:10.1002/jor. 1100150404

Shirazi R, Shirazi-Adl A (2008) Deep vertical collagen fibrils play a significant role in mechanics of articular cartilage. J Orthop Res 26(5):608-615. doi:10.1002/jor.20537

Shirazi R, Shirazi-Adl A, Hurtig M (2008) Role of cartilage collagen fibrils networks in knee joint biomechanics under compression. J Biomech 41(16):3340-3348. doi:10.1016/j.jbiomech.2008.09. 033

Van Turnhout MC, Haazelager MB, Gijsen MA, Schipper H, Kranenbarg S, Van Leeuwen JL (2008) Quantitative description of collagen structure in the articular cartilage of the young and adult equine distal metacarpus. Anim Biol 58(4):353-370. doi:10.1163/ $157075608 \times 383674$

Van Turnhout MC, Schipper H, Engel B, Buist W, Kranenbarg S, Van Leeuwen JL (2010) Postnatal development of collagen structure in ovine articular cartilage. BMC Dev Biol (In press)

Wang CCB, Guo XE, Sun D, Mow VC, Ateshian GA, Hung CT (2002) The functional environment of chondrocytes within cartilage subjected to compressive loading: a theoretical and experimental approach. Biorheology 39(1-2):11-25

Williamson AK, Chen AC, Sah RL (2001) Compressive properties and function-composition relationships of developing bovine articular cartilage. J Orthop Res 19(6):1113-1121. doi:10.1016/ S0736-0266(01)00052-3 
Wilson W, van Donkelaar C, van Rietbergen B, Ito K, Huiskes R (2004) Stresses in the local collagen network of articular cartilage: a poroviscoelastic fibril-reinforced finite element study. J Biomech 37(3):357-366 doi:10.1016/S0021-9290(03)00267-7, erratum in J Biomech 38(10):2138-2140, see Wilson et al. (2005b)

Wilson W, van Donkelaar C, van Rietbergen B, Huiskes R (2005a) A fibril-reinforced poroviscoelastic swelling model for articular cartilage. J Biomech 38(6):1195-1204, doi:10.1016/j.jbiomech.2004. 07.003, erratum in J Biomech 38(10):2138-2140, see Wilson et al (2005b)

Wilson W, van Donkelaar C, van Rietbergen B, Ito K, Huiskes R (2005b) Erratum to "Stresses in the local collagen network of articular cartilage: a poroviscoelastic fibril-reinforced finite element study" [J Biomech 37 (2004) 357-366] and "A fibril-reinforced poroviscoelastic swelling model for articular cartilage" [J Biomech 38 (2005) 1195-1204]. J Biomech 38(10):2138-2140. doi:10.1016/j.jbiomech.2005.04.024

Wilson W, Driessen N, Van Donkelaar C, Ito K (2006) Prediction of collagen orientation in articular cartilage by a collagen remodeling algorithm. Osteoarthritis Cartilage 14(11):1196-1202. doi:10. 1016/j.joca.2006.05.006

Wilson W, Huyghe J, van Donkelaar C (2006) A composition-based cartilage model for the assessment of compositional changes during cartilage damage and adaptation. Osteoarthritis Cartilage 14(6):554-560. doi:10.1016/j.joca.2005.12.006

Wilson W, Huyghe J, van Donkelaar C (2007) Depth-dependent compressive equilibrium properties of articular cartilage explained by its composition. Biomech Model Mechanobiol 6(1-2):43-53. doi:10.1007/s10237-006-0044-z

Wong M, Wuethrich P, Buschmann M, Eggli P, Hunziker E (1997) Chondrocyte biosynthesis correlates with local tissue strain in statically compressed adult articular cartilage. J Orthop Res 15(2):189-196. doi:10.1002/jor.1100150206

Wong M, Ponticiello M, Kovanen V, Jurvelin J (2000) Volumetric changes of articular cartilage during stress relaxation in unconfined compression. J Biomech 33(9):1049-1054. doi:10.1016/ S0021-9290(00)00084-1

Wu JZ, Herzog W (2006) Analysis of the mechanical behavior of chondrocytes in unconfined compression tests for cyclic loading. J Biomech 39(4):603-616. doi:10.1016/j.jbiomech.2005.01.007

Zambrano NZ, Montes GS, Shigihara KM, Sanchez EM, Junqueira LC (1982) Collagen arrangement in cartilages. Acta Anat 113(1):26-38. doi:10.1159/000145534

Zheng Y, Niu H, Arthur Mak F, Huang Y (2005) Ultrasonic measurement of depth-dependent transient behaviors of articular cartilage under compression. J Biomech 38(9):1830-1837. doi:10.1016/j. jbiomech.2004.08.020 